\title{
Bioinformatic analysis of gene expression profiling of intracranial aneurysm
}

\author{
LIJUAN BO ${ }^{1 *}, \mathrm{BO} \mathrm{WEI}^{2 *}, \mathrm{ZHANFENG} \mathrm{WANG}^{2}, \mathrm{CHAOHUI} \mathrm{LI}^{2}, \mathrm{ZHENG} \mathrm{GAO}^{3}$ and ZHUANG MIAO $^{2}$ \\ Departments of ${ }^{1}$ Infection and ${ }^{2}$ Neurosurgery, China-Japan Union Hospital of Jilin University, Changchun, \\ Jilin 130033; ${ }^{3}$ Department of Neurosurgery, Dandong First Hospital, Dandong, Liaoning 118000, P.R. China
}

Received May 19, 2016; Accepted April 4, 2017

DOI: $10.3892 / \mathrm{mmr} .2017 .8367$

\begin{abstract}
Intracranial aneurysm (IA) is a severe clinical condition of primary concern and currently, there is no effective therapeutic reagent. The present study aimed to investigate the molecular mechanism of IA via bioinformatic analysis. Various gene expression profiles (GSE26969) were downloaded from the Gene Expression Omnibus database, including 3 IA and 3 normal superficial temporal artery samples. Firstly, the limma package in R language was used to identify differentially expressed genes (DEGs; P-value $<0.01$ and $\mid \log _{2} F C l \geq 1$ ). Secondly, the database for annotation, visualization and integrated discovery software was utilized to perform pathway and functional enrichment analyses (false discovery rate $\leq 0.05$ ). Finally, protein-protein interaction (PPI) network and sub-network clustering analyses were performed using the biomolecular interaction network database and ClusterONE software, respectively. Following this, a transcription factor regulatory network was identified from the PPI network. A total of 1,124 DEGs were identified, of which 989 were upregulated and 135 downregulated. Pathway and functional enrichment analyses revealed that the DEGs primarily participated in RNA splicing, functioning of the spliceosome, RNA processing and the mRNA metabolic process. Following PPI network analysis, 1 hepatocyte nuclear factor $(H N F) 4 A$ (transcription factor)-centered regulatory network and 5 DEG-centered sub-networks were identified. On analysis of the transcription factor regulatory network, 6 transcription factors (HNF6, HNF4A, E2F4, YY1, H4 and $H 31 T$ ) and a regulatory pathway (HNF6-HNF4-E2F4) were identified. The results of the present study suggest that
\end{abstract}

Correspondence to: Dr Zhuang Miao, Department of Neurosurgery, China-Japan Union Hospital of Jilin University, 126 Xiantai Street, Changchun, Jilin 130033, P.R. China

E-mail: miaozhzhzhuuuu@hotmail.com

*Contributed equally

Key words: intracranial aneurysm, differentially expressed genes, functional enrichment analysis, protein-protein interaction, regulatory network activating transcription factor-5, Jun proto-oncogene, activator protein-1 transcription factor subunit, $H N F 6, H N F 4$ and $E 2 F 4$ may participate in IA progression via vascular smooth muscle cell apoptosis, inflammation, vessel wall remodeling and damage and the tumor necrosis factor- $\beta$ signaling pathway. However, further experimental studies are required to validate these predictions.

\section{Introduction}

Intracranial aneurysm (IA) is a cerebrovascular disorder characterized by a regional ballooning of intracranial arteries. IAs are classified into saccular aneurysms, fusiform aneurysms, and micro-aneurysms according to their size and shape. IA is a frequently occurring condition with a prevalence of $2-5 \%$ in the global population, and $\sim 0.7-1.9 \%$ of cases transform into subarachnoid hemorrhage (SAH) (1). $\mathrm{SAH}$, which constitutes $1-7 \%$ of all strokes, has high mortality and morbidity rates, even though diagnosis and treatment with modern medical technologies are available (2). Currently, imaging methods including digital subtraction angiography, computed tomography (CT) and CT angiography are the primary IA diagnostic methods, however there is no available preventive treatment of IA prior to rupture, except for surgical procedures and endovascular therapy. However, these treatment methods often result in further complications. The requirement to develop novel therapeutic strategies for IA is of primary concern, and understanding the underlying molecular mechanism of its initiation will aid this research.

Recently, numerous studies have been conducted to identify the pathogenesis of IA formation and rupture. Based on affymetrix microarray data from 3 unruptured IAs and a control superficial temporal artery, Li et al (3) demonstrated that upregulated genes are extracellular matrix (ECM)-associated genes and downregulated genes are enriched in the immune/inflammation response. Based on data obtained from 8 ruptured IAs, 6 unruptured IAs and 5 control intracranial arteries, Pera et al (4) demonstrated that upregulated genes are enriched in the immune/inflammatory response and downregulated genes are enriched in the muscle system and cell adhesion. Based on data from 11 ruptured IAs and 8 unruptured IAs, Kurki et al (5) demonstrated that upregulated genes are enriched in chemotaxis, leukocyte migration, oxidative stress, vascular remodeling, ECM degradation and 
the response to turbulent blood flow. In addition, Yagi et al (6) demonstrated that phosphodiesterase-4 is involved in inflammatory diseases and blocking it reduces macrophage migration and inhibits the progression of cerebral aneurysms (CA). Aoki et al (7) revealed that v-ets avian erythroblastosis virus E26 oncogene homolog EST-1 is over-expressed in vascular smooth muscle cells (VSMCs) in CA walls, and thus promotes CA progression.

The present study firstly downloaded gene expression profiling data. The raw data were then analyzed to identify differentially expressed genes (DEGs) between IA and normal superficial temporal artery tissue. Following this, pathway and functional enrichment analyses were conducted to investigate the functions of the identified DEGs. A protein-protein interaction (PPI) network was then constructed and visualized, from which a transcription factor regulatory network was identified.

\section{Materials and methods}

Obtaining and preprocessing of microarray data. Gene expression profiles of IA and normal superficial temporal artery samples were obtained from the National Center of Biotechnology Information (NCBI) Gene Expression Omnibus (GEO; www.ncbi.nlm.nih.gov/geo/) database. The access number is GSE26969, and this dataset included a total of 6 samples; 3 IA and 3 normal superficial temporal artery samples. The platform used was the Affymetrix Human Genome U133 Plus 2.0 Array (GPL570; Affymetrix; Thermo Fisher Scientific, Inc., Waltham, MA, USA). Firstly, the original data at the probe symbol level were converted into expression values at the gene symbol level. For each sample, the expression values of all probes mapped to a given gene symbol were averaged. Then, missing data were inserted and median data normalization performed by using the robust multichip averaging (RMA) method (8).

DEG screening and clustering analysis. The limma package (http://www.bioconductor.org/packages/2.9/bioc /html/limma.html) (9) in R language with multiple testing correction based on the Benjamini \& Hochberg method (10) was employed to identify DEGs between IA and normal superficial temporal artery samples. P-value $<0.01$ and $\log _{2}$ fold change (FC) $I \geq 1$ were used as the thresholds for this analysis. Then, the pheatmap package (https://cran.rproject. org/web/packages/pheatmap/index.html) (11) in R language was used to cluster the identified DEGs.

Enrichment analysis of DEGs. To study DEGs at a functional level, GO (Gene Ontology) functional enrichment analysis (12) and KEGG (Kyoto Encyclopedia of Gene and Genomes) pathway enrichment (13) analysis were performed using the online biological tool, database for the annotation, visualization and integrated discovery (DAVID version 6.7; https://david-d. ncifcrf.gov/) (14). DAVID software has been extensively used to identify biological processes involving a given list of genes. In the present study, fold change discovery (FDR) $\leq 0.05$ was set as the cut-off criterion for enrichment analysis.

PPI network construction. PPIs are important for all biological processes. The present study constructed a PPI network
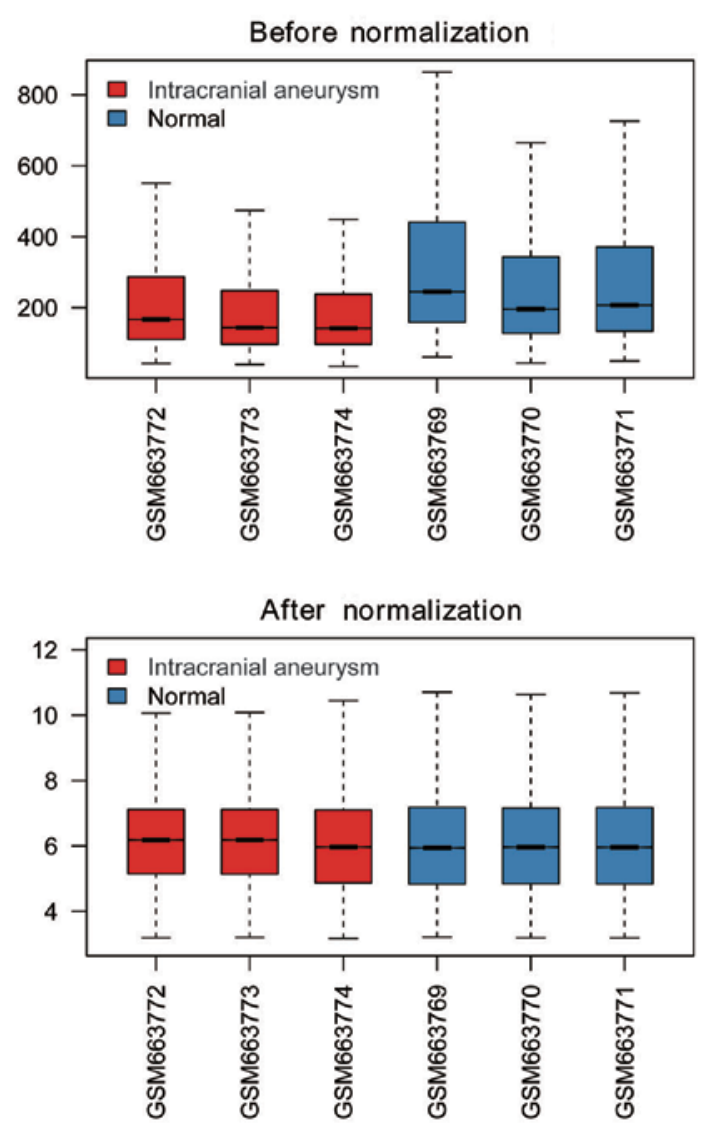

Figure 1. Boxplot of data prior to and following normalizationn.

among DEGs based on the biomolecular interaction network (BIND) database (http://binddb.org) (15). BIND is available for querying, viewing and submitting records regarding molecular interactions, complexes and pathway information. Additionally, ClusterONE software (http://www.paccanarolab. org/cluster-one/) (16) was used to perform sub-network clustering analysis and then GO functional enrichment analysis was then performed for DEGs in each sub-network.

\section{Results}

Data preprocessing and DEG screening. Data prior to and following normalization are presented in Fig. 1. Following normalization, gene expression values of differing samples ranged almost uniformly, which indicated that all of the 6 chips were available for further analysis. Following DEG screening using the limma package, a total of 1124 DEGs $\left(\mid \log _{2} \mathrm{FCl} \geq 1\right.$ and P-value $\leq 0.01$ ) were identified, of which 989 DEGs were upregulated and 135 DEGs downregulated (Fig. 2A). In addition, the top 5 upregulated and top 5 downregulated DEGs are presented in Table I.

Clustering analysis. A clustering analysis was performed using the pheatmap package in R language and 1124 DEGs were divided into two major clusters (Fig. 2B). The DEGs in the upper cluster were significantly downregulated, whereas the DEGs in the lower cluster were significantly downregulated. The separation of the clusters into two differing categories indicated that the identified DEGs were significantly differentially 
A

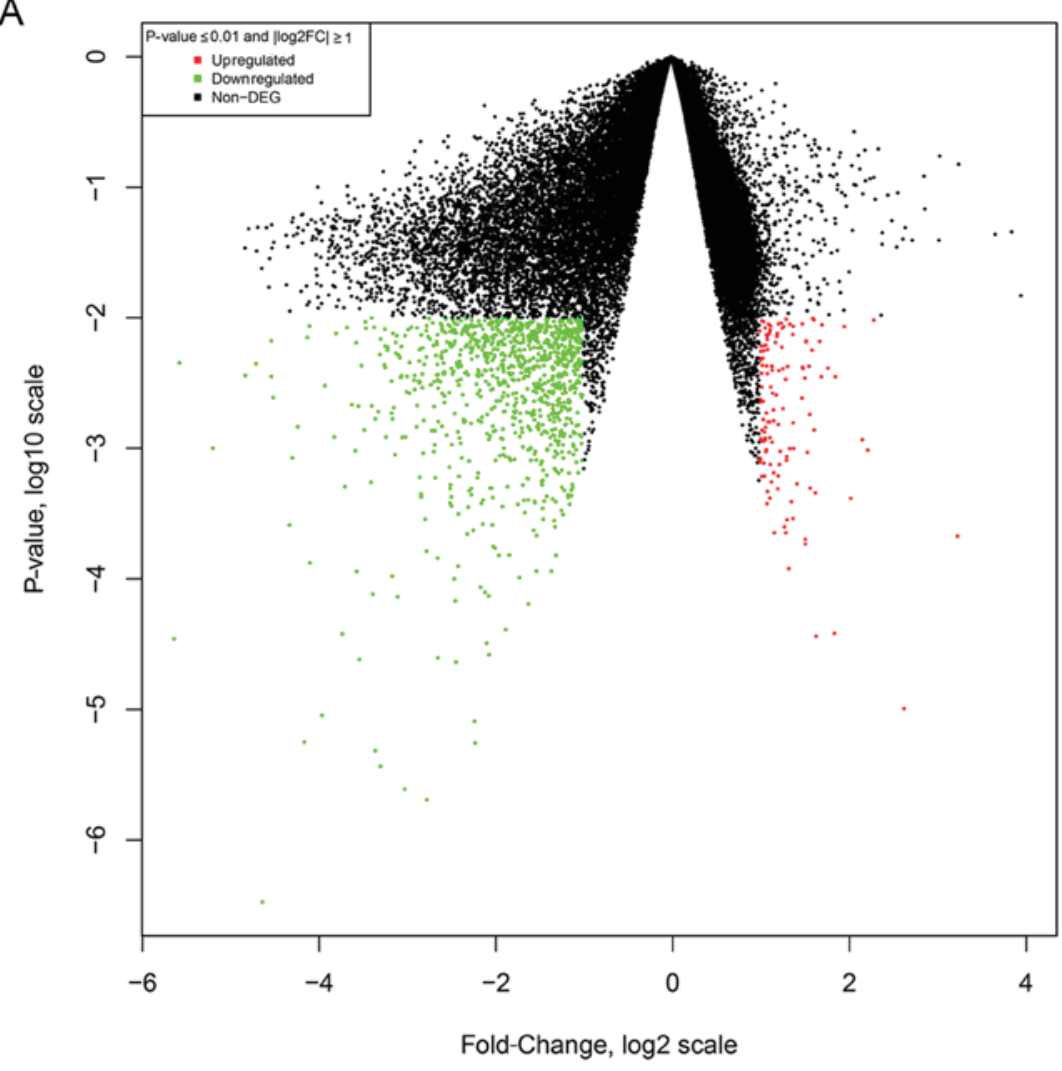

B

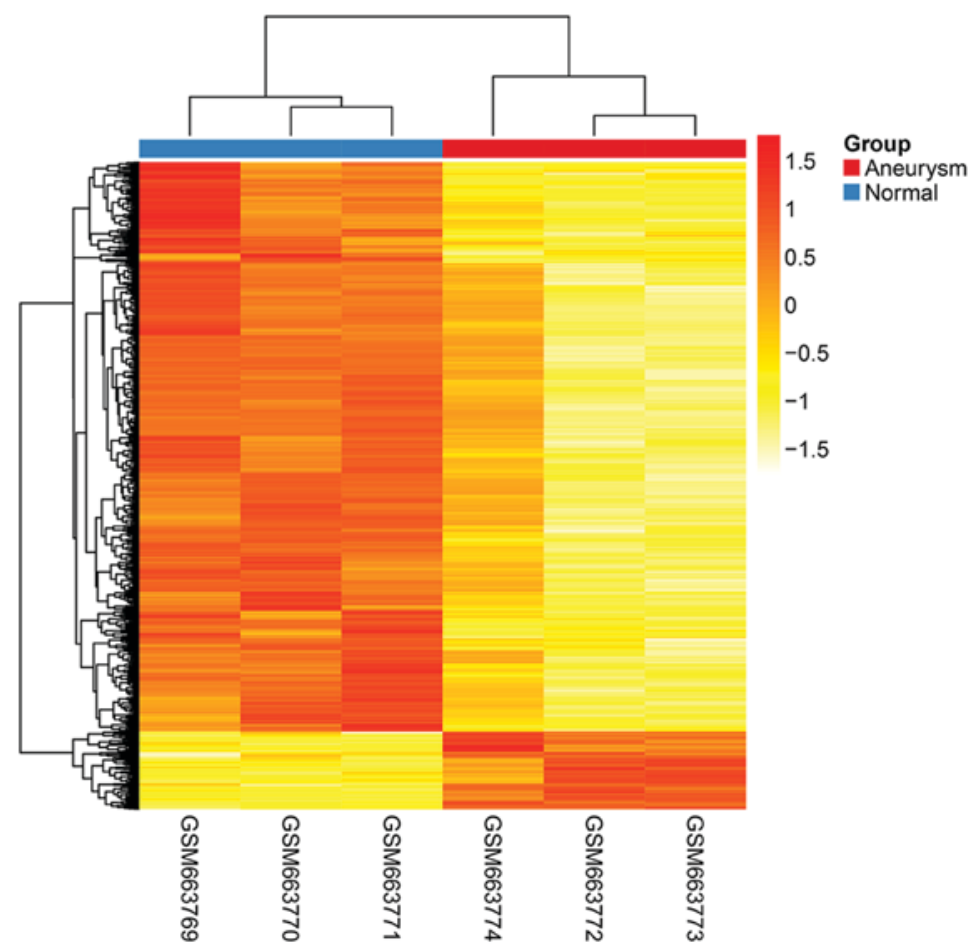

Figure 2. Volcano plot and heatmap of differentially expressed genes. (A) Volcano plot and (B) heatmap of differentially expressed genes following clustering analysis.

expressed between IA and normal superficial temporal artery samples.

Enrichment analysis of DEGs. KEGG pathway and GO functional enrichment analyses demonstrated that 1124 DEGs were enriched in 1 KEGG pathway (hsa03040: spliceosome, FDR $\leq 0.05$ ) and $20 \mathrm{GO}$ functions (FDR $\leq 0.05$; Fig. 3 ). These GO functions were primarily associated with RNA splicing and the spliceosome, RNA processing and the mRNA metabolic process. 
Table I. Top 5 upregulated and downregulated differentially expressed genes.

\begin{tabular}{|c|c|c|c|c|}
\hline Gene & Average expression value & $\log _{2} \mathrm{FC}$ & P-value & Expression alteration \\
\hline$P L N$ & 8.622363 & -5.62222 & $3.47 \times 10^{-5}$ & Downregulated \\
\hline$A D H 1 C$ & 8.253548 & -5.56344 & $4.51 \times 10^{-3}$ & Downregulated \\
\hline$P L N$ & 7.481133 & -5.18628 & $1.00 \times 10^{-3}$ & Downregulated \\
\hline MYL9 & 9.273575 & -4.81707 & $3.61 \times 10^{-3}$ & Downregulated \\
\hline SORBS1 & 8.440836 & -4.69688 & $4.44 \times 10^{-3}$ & Downregulated \\
\hline MMP16 & 7.516438 & 2.166793 & $1.17 \times 10^{-3}$ & Upregulated \\
\hline SOX4 & 9.123515 & 2.227376 & $9.69 \times 10^{-4}$ & Upregulated \\
\hline NUFIP2 & 8.381299 & 2.294707 & $9.57 \times 10^{-3}$ & Upregulated \\
\hline TWISTI & 6.786085 & 2.636303 & $1.01 \times 10^{-5}$ & Upregulated \\
\hline COL5A2 & 10.29836 & 3.242704 & $2.12 \times 10^{-4}$ & Upregulated \\
\hline
\end{tabular}

FC, fold change.

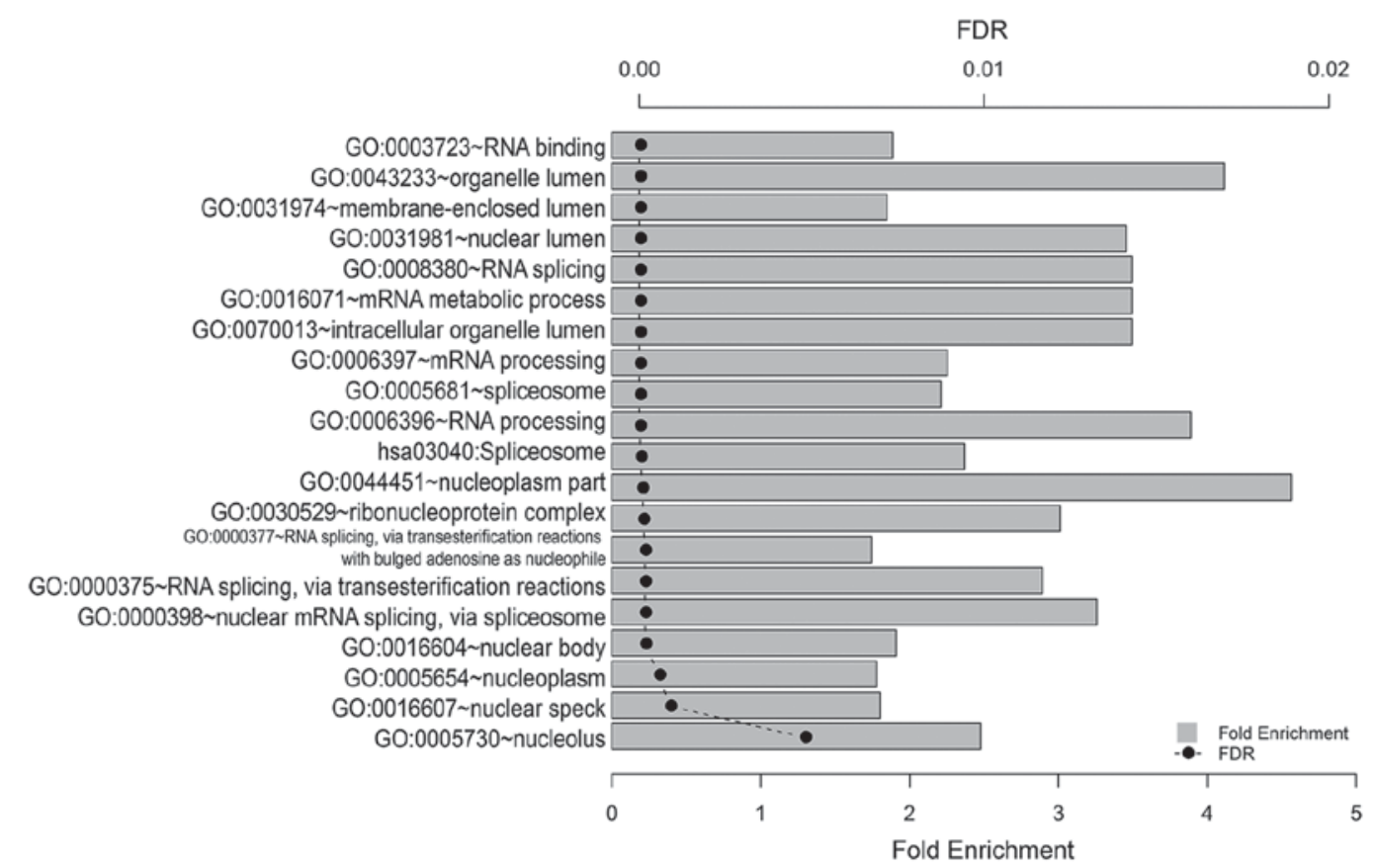

Figure 3. Enrichment analysis of differentially expressed genes. FDR, false discovery rate; GO, gene ontology.

PPI network construction. A PPI network was then constructed using the BIND database (Fig. 4), in which a hepatocyte nuclear factor $(H N F) 4 A$ (transcription factor)-centered regulatory network was identified. In addition, a sub-network clustering analysis was performed using ClusterONE software and 5 DEG-centered sub-networks were identified (Fig. 5A-E) and significantly enriched in $3 \mathrm{GO}$ functions (Table II). The central DEGs observed in the sub-networks were origin recognition complex subunit $2(O R C 2 L)$, activating transcription factor 5 (ATF5), MAF BZIP transcription factor $\mathrm{K}(M A F K)$ and Jun proto-oncogene, activator protein-1 transcription factor subunit (JUN).

Transcription factor regulatory network construction. The interactions between DEGs and transcription factors were identified from the PPI network, and presented in Fig. 6. A total of 6 transcription factors (HNF6, HNF4A, E2F4, YY1, H4 and $H 31 T$ ) and 24 DEGs (5 upregulated and 19 downregulated genes) were involved in the transcription factor regulatory network. It was observed that $H N F 4 A$ exhibited the ability to regulate the DEGs, including prolylcarboxypeptidase (PRCP) and caspase- 8 and Fas associated via death domain-like apoptosis regulator (CFLAR).

\section{Discussion}

IA is a severe clinical condition and as of yet, there is no current effective therapy. In the present study, bioinformatic analyses were conducted to investigate the potential molecular mechanism underlying the occurrence of IA. Consequently, 1,124 DEGs were identified between IA and normal superficial temporal artery tissue. The DEGs primarily participated 


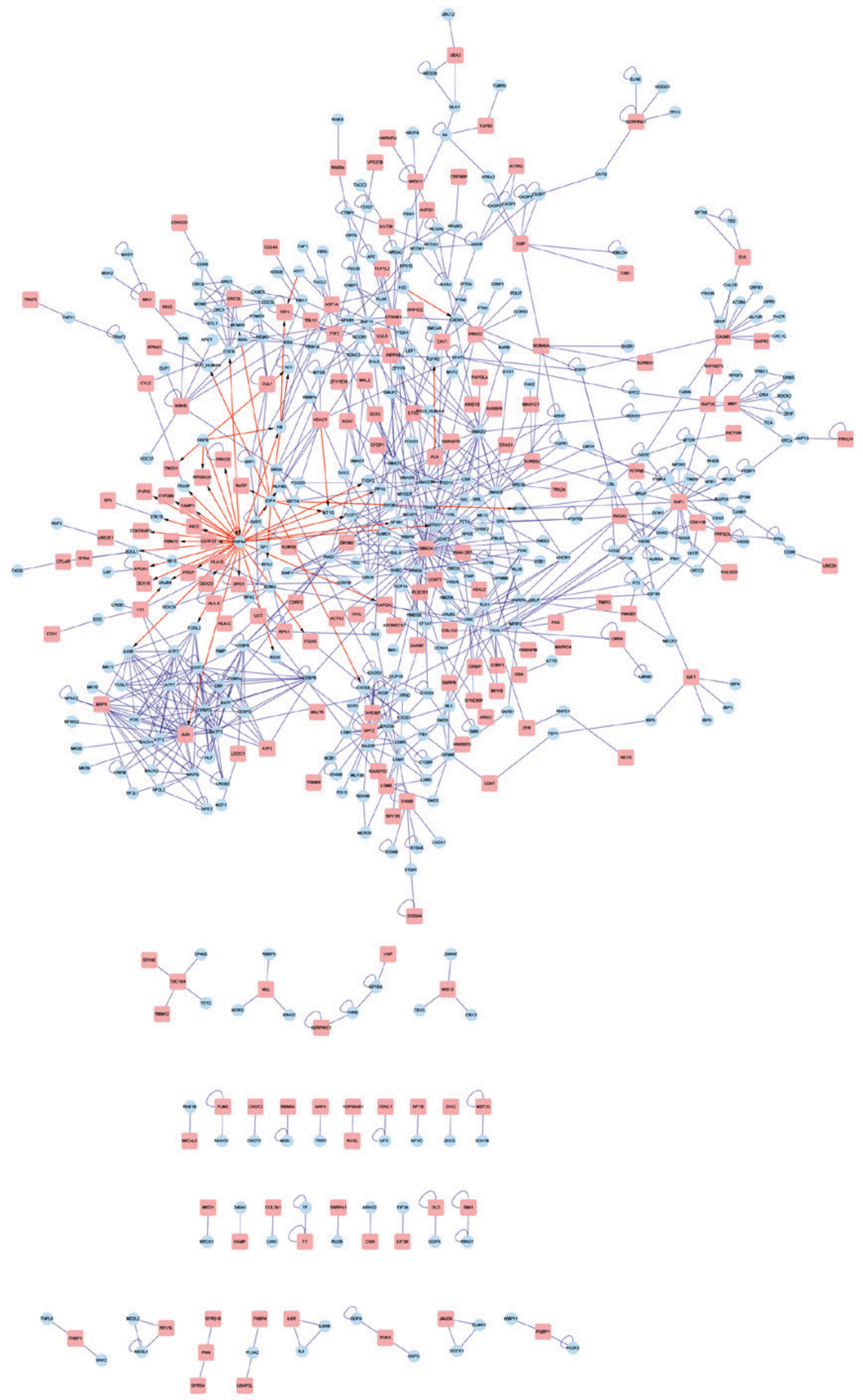

Figure 4. Protein-protein interaction network. A representative figure demonstrating the large scale and connectivity of the protein-protein interaction network. Red squares represent DEGs and blue circles represent genes that interact with the DEGs. Red arrows represent interactions between transcription factors and target genes and blue lines represent interactions between genes. DEGS, differentially expressed genes. 
Table II. Sub-networks from protein-protein interaction network.

\begin{tabular}{|c|c|c|c|c|c|}
\hline & Sub-network 1 & Sub-network 2 & Sub-network 3 & Sub-network 4 & Sub-network 5 \\
\hline Nodes & 8 & 14 & 8 & 15 & 15 \\
\hline Density & 0.679 & 0.615 & 0.607 & 0.6 & 0.6 \\
\hline Quality & 0.792 & 0.560 & 0.708 & 0.525 & 0.496 \\
\hline $\mathrm{P}$-value & $4.552 \times 10^{-4}$ & $5.069 \times 10^{-4}$ & $5.367 \times 10^{-4}$ & $1.00 \times 10^{-3}$ & $3.00 \times 10^{-3}$ \\
\hline \multirow[t]{15}{*}{ Genes } & MCM10 & CEBPE & $C D K 6$ & $M A F K$ & $M A F K$ \\
\hline & $M C M 2$ & DDIT3 & MCM10 & $J U N$ & $J U N$ \\
\hline & CDC6 & $C E B P G$ & MCM2 & DDIT3 & DDIT3 \\
\hline & $O R C 2 L$ & $C E B P B$ & CDC6 & $C E B P G$ & CEBPG \\
\hline & ORC1 & $D B P$ & $O R C 2 L$ & $M A F G$ & $M A F G$ \\
\hline & ORC5 & $C E B P D$ & ORC1 & CREB3 & NFAC2 \\
\hline & $C D C 5 L$ & ATF5 & ORC5 & FOSL1 & FOSL1 \\
\hline & ORC4 & CEBPA & ORC4 & FOS & FOS \\
\hline & & CREB3 & & FOSL2 & FOSL2 \\
\hline & & $H L F$ & & ATF3 & ATF3 \\
\hline & & ATF3 & & $J U N B$ & $J U N B$ \\
\hline & & $B A T F 3$ & & $B A T F 3$ & $B A T F 3$ \\
\hline & & ATF7 & & $A T F 7$ & ATF7 \\
\hline & & $B A T F$ & & $A T F 2$ & $A T F 2$ \\
\hline & & & & BACH2 & $B A T F$ \\
\hline $\begin{array}{l}\text { Top significant } \\
\text { GO term }\end{array}$ & $\begin{array}{c}\text { GO:0006260 } \\
\text { DNA replication }\end{array}$ & $\begin{array}{c}\text { GO:0046983 } \\
\text { protein dimerization } \\
\text { activity }\end{array}$ & $\begin{array}{c}\text { GO:0006260 } \\
\text { DNA replication }\end{array}$ & $\begin{array}{l}\text { GO:0043565 } \\
\text { sequence-specific } \\
\text { DNA binding }\end{array}$ & $\begin{array}{c}\text { GO:0043565 } \\
\text { sequence-specific } \\
\text { DNA binding }\end{array}$ \\
\hline
\end{tabular}

GO, gene ontology.

A

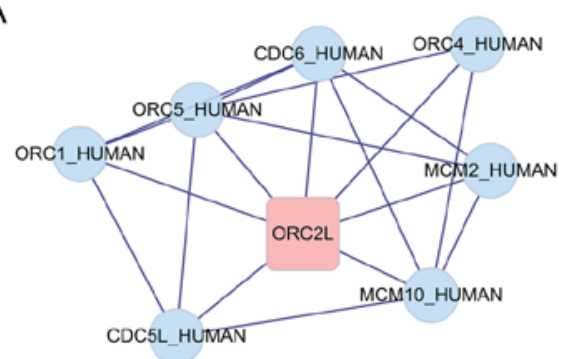

D

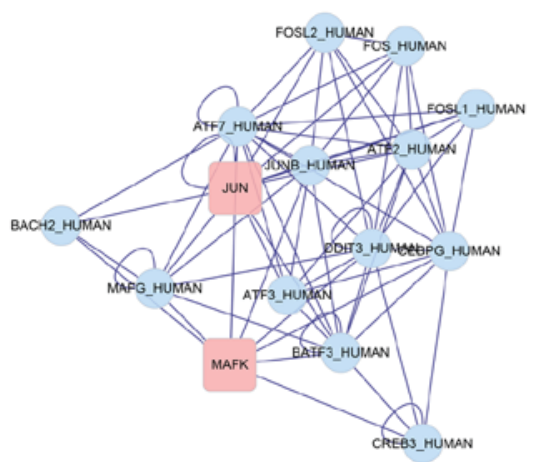

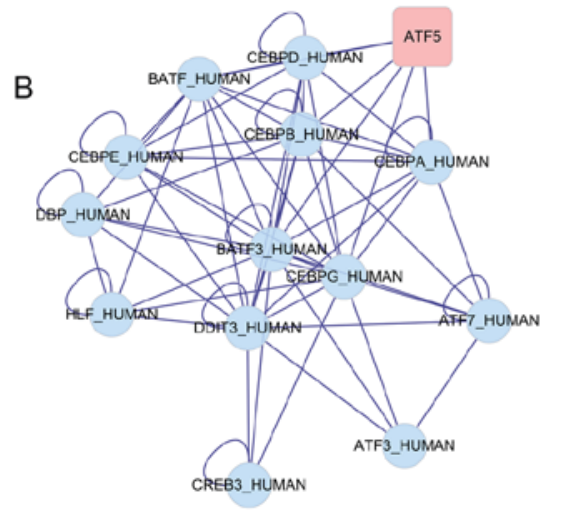
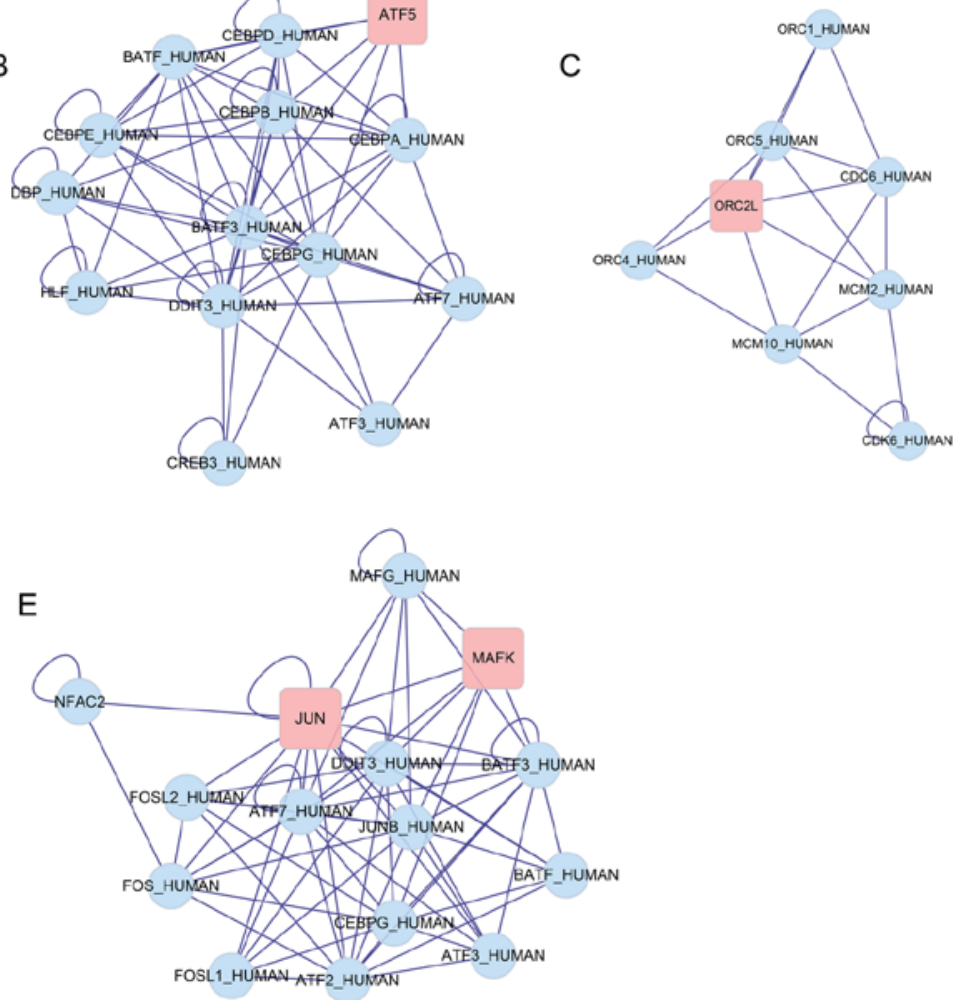

Figure 5. Sub-networks of differentially expressed genes extracted from the protein-protein interaction network. Sub-networks (A) 1, (B) 2, (C) 3, (D) 4 and (E) 5. Red squares represent DEGs, blue circles represent genes that interact with the DEGs and blue lines represent interactions between genes. DEGs, differentially expressed genes. 


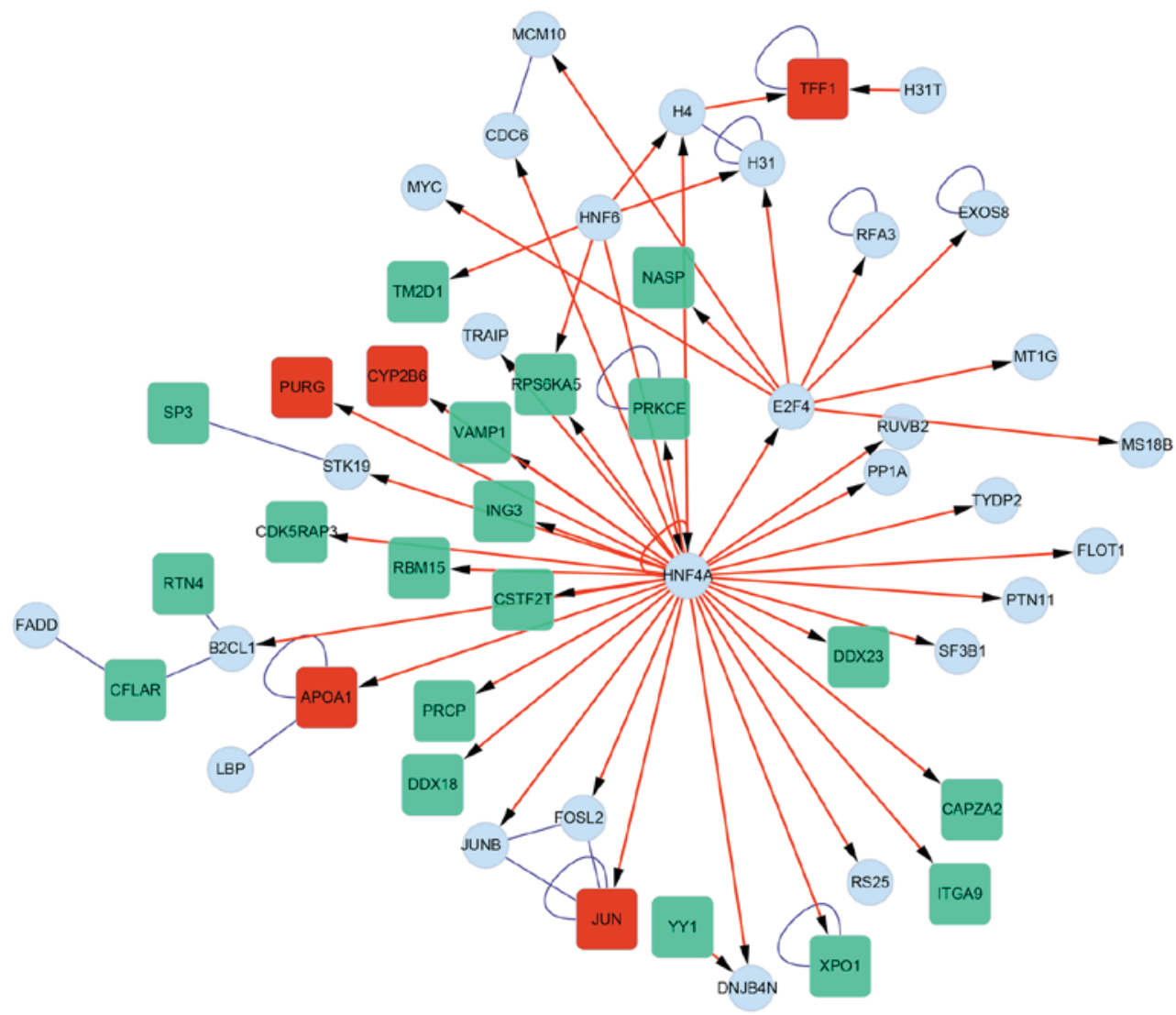

Figure 6. Transcription factor regulatory network. Red squares represent upregulated DEGs, green squares represent downregulated DEGs and blue circles represent genes that interact with the DEGs. Red arrows represent interactions between transcription factors and target genes and blue lines represent interactions between genes. DEGs, differentially expressed genes.

in RNA splicing and the spliceosome, RNA processing, and mRNA metabolic processing. Following this, a PPI network was constructed and 5 DEG-centered sub-networks were revealed, involving 4 DEGs $(O R C 2 L, A T F 5, M A F K$ and $J U N)$. Furthermore, a transcription factor regulatory network was abstracted, and 6 transcription factors (HNF6, HNF4A, E2F4, YYl, H4 and H3IT) and 24 DEGs (including PRCP and CFLAR) were involved.

From the results of the sub-pathway clustering analysis, the PPI network was divided into 5 DEG-centered sub-networks, involving 4 DEGs $(O R C 2 L, A T F 5, M A F K$ and $J U N)$. Various DEGs identified included ATF5 (sub-network 2), which is a stress-response transcription factor and is highly expressed in various tumors (17). CCAAT/enhancer binding protein $\beta$ $(C E B P B)$ interacts with $A T F 5$ and encodes a basic-region leucine zipper transcription factor (bZIP). $C E B P B$ participates in the regulation of the immune and inflammatory response, and thus may participate in the pathogenesis of IA $(18,19)$. Therefore, ATF5 may participate in IA via its interaction with $C E B P B$. Furthermore, $J U N$ (sub-network 4 and 5) is a bZIP transcription factor involved in numerous cellular processes, such as cell growth and apoptosis (20), and tumor necrosis factor (TNF)- $\alpha$ has been demonstrated to stimulate prolonged activation of $J U N$ and interleukin (IL)-1 $\beta$ gene expression (21). TNF- $\alpha$ is known to activate matrix metalloproteinase which is important in IA pathology $(22,23)$. In addition, Moriwaki et al (24) demonstrated that IL-1 $\beta$ is important for the progression of IA via the induction of VSMC apoptosis. Therefore, JUN may participate in the progression of IA by responding to TNF- $\alpha$ and IL-1 $\beta$.

The HNF4A-centered transcription factor regulatory network led to the identification of the regulatory pathway HNF6-HNF4A-E2F4. HNF6 encodes a transcription factor that has been demonstrated to influence a variety of cellular processes, including cell proliferation, cell-matrix adhesion and inflammation (25). The pathological pathway for IA involves endothelial dysfunction/injury, the mounting inflammatory response, VSMC phenotypic modulation, extra-cellular matrix remodeling, subsequent cell death and vessel wall degeneration (26). Therefore, HNF6 may participate in IA via induction of inflammation. HNF4A encodes a transcription factor that participates in lipid metabolic processes. Frösen et al (27) demonstrated that accumulation of lipids and oxidation in the saccular intracranial aneurysm (sIA) wall are associated with its degeneration, resulting in fatal SAH (27). Therefore, HNF4A may participate in the progression of IA via regulation of vessel wall remodeling and damage. Furthermore, E2F4 encodes E2F transcription factor 4 which participates in the cell cycle and tumor suppression. E2F4 participates in the transforming growth factor (TGF) $-\beta$ signaling pathway which is involved in cell differentiation, cell growth and apoptosis. It has previously been reported that the TGF- $\beta$ signaling pathway is important in IA (28). Therefore, $E 2 F 4$ may be involved in IA progression via its participation in the TGF- $\beta$ signaling pathway. 
Of the DEGs regulated by HNF4A, PRCP encodes an enzyme which cleaves $\mathrm{COOH}$-terminal amino acids linked to proline, in peptides including angiotension II (29). Angiotensin II, a part of the renin-angiotensin system (RAS), is a potent vasoconstrictor and pro-inflammatory stimulant. Shoja et al (30) demonstrated that RAS is associated with the pathogenesis of IA. Therefore, $P R C P$ was predicted to participate in IA progression via the regulation of RAS. In addition, CFLAR encodes the caspase- 8 and Fas associated via death domain-like apoptosis regulator (31), involved in the TNF- $\alpha$ /nuclear factor (NF)- $\kappa B$ signaling pathway. NF- $\kappa B$ recruits macrophages into the vessel walls involved in IA, initiating inflammation and participating in aneurysm formation (32). Therefore, CFLAR was hypothesized to participate in IA progression via the TNF- $\alpha / \mathrm{NF}-\kappa \mathrm{B}$ signaling pathway.

In conclusion, the results of the present study revealed that inflammation may be an important factor in AI development. In order to elucidate the pathology of IA, gene expression profiling data of 6 samples were downloaded and analyzed. Following a sub-network clustering analysis, 5 sub-networks were identified, involving 4 DEGs (ORC2L, ATF5, MAFK and JUN). ATF5 and $J U N$ served crucial roles in the immune and inflammatory response and VSMC apoptosis during AI development. In addition, the regulatory pathway HNF6-HNF4-E2F4 was identified as an important mechanism involved in the development of AI via the regulation of inflammation, the TGF- $\beta$ signaling pathway, the renin-angiotensin system and the TNF- $\alpha / N F-\kappa B$ signaling pathway. However, these results were derived from in silico analysis; thus, further experimental studies are required to verify the results of the present study.

\section{References}

1. Alg VS, Sofat R, Houlden H and Werring DJ: Genetic risk factors for intracranial aneurysms: A meta-analysis in more than 116,000 individuals. Neurology 80: 2154-2165, 2013.

2. Bakke SJ and Lindegaard KF: Subarachnoid haemorrhage-diagnosis and management. Tidsskr Nor Laegeforen 127: 1074-1078, 2007 (In Norwegian).

3. Li L, Yang X, Jiang F, Dusting GJ and Wu Z: Transcriptome-wide characterization of gene expression associated with unruptured intracranial aneurysms. Eur Neurol 62: 330-337, 2009.

4. Pera J, Korostynski M, Krzyszkowski T, Czopek J, Slowik A, Dziedzic T, Piechota M, Stachura K, Moskala M, Przewlocki R and Szczudlik A: Gene expression profiles in human ruptured and unruptured intracranial aneurysms: What is the role of inflammation? Stroke 41: 224-231, 2010

5. Kurki MI, Hakkinen SK, Frosen J, Tulamo R, von und zu Fraunberg M, Wong G, Tromp G, Niemelä M, Hernesniemi J, Jääskeläinen JE and Ylä-Herttuala S: Upregulated signaling pathways in ruptured human saccular intracranial aneurysm wall: An emerging regulative role of Toll-like receptor signaling and nuclear factor- $\kappa \mathrm{B}$, hypoxia-inducible factor-1A and ETS transcription factors. Neurosurgery 68: 1666-1675, 2011.

6. Yagi K, Tada Y, Kitazato KT, Tamura T, Satomi J and Nagahiro S: Ibudilast inhibits cerebral aneurysms by down-regulating inflammation-related molecules in the vascular wall of rats. Neurosurgery 66 : 551-559, 2010.

7. Aoki T, Kataoka H, Nishimura M, Ishibashi R, Morishita R and Miyamoto S: Ets-1 promotes the progression of cerebral aneurysm by inducing the expression of MCP-1 in vascular smooth muscle cells. Gene Ther 17: 1117-1123, 2010.

8. Irizarry RA, Hobbs B, Collin F, Beazer-Barclay YD, Antonellis KJ, Scherf U and Speed TP: Exploration, normalization and summaries of high density oligonucleotide array probe level data. Biostatistics 4: 249-264, 2003.

9. Gentleman RC, Carey VJ, Bates DM, Bolstad B, Dettling M, Dudoit S, Ellis B, Gautier L, Ge Y, Gentry J et al: Bioconductor: Open software development for computational biology and bioinformatics. Genome Biol 5: R80, 2004.
10. Reiner A, Yekutieli D and Benjamini Y: Identifying differentially expressed genes using false discovery rate controlling procedures. Bioinformatics 19: 368-375, 2003.

11. Kolde R: Pheatmap: Pretty heatmaps. R package version 0.6 1: 2012.

12. Ashburner M, Ball CA, Blake JA, Botstein D, Butler H, Cherry JM, Davis AP, Dolinski K, Dwight SS, Eppig JT, et al: Gene ontology: Tool for the unification of biology. The Gene Ontology Consortium. Nat Genet 25: 25-29, 2000.

13. Kanehisa M and Goto S: KEGG: kyoto encyclopedia of genes and genomes. Nucleic Acids Res 28: 27-30, 2000.

14. Huang da W, Sherman BT and Lempicki RA: Systematic and integrative analysis of large gene lists using DAVID bioinformatics resources. Nat Protoc 4: 44-57, 2009.

15. Bader GD, Betel D and Hogue CW: BIND: The biomolecular interaction network database. Nucleic Acids Res 31: 248-250, 2003.

16. Nepusz T, Yu H and Paccanaro A: Detecting overlapping protein complexes in protein-protein interaction networks. Nat Methods 9: 471-472, 2012.

17. Greene LA, Lee HY and Angelastro JM: The transcription factor ATF5: Role in neurodevelopment and neural tumors. J Neurochem 108: 11-22, 2009.

18. Schoenen H, Huber A, Sonda N, Zimmermann S, Jantsch J, Lepenies B, Bronte V and Lang R: Differential control of mincle-dependent cord factor recognition and macrophage responses by the transcription factors C/EBP $\beta$ and HIF1 $\alpha$. J Immunol 2014.

19. Chalouhi N, Ali MS, Jabbour PM, Tjoumakaris SI, Gonzalez LF, Rosenwasser RH, Koch WJ and Dumont AS: Biology of intracranial aneurysms: Role of inflammation. J Cereb Blood Flow Metab 32: 1659-1676, 2012.

20. Wisdom R, Johnson RS and Moore C: c-Jun regulates cell cycle progression and apoptosis by distinct mechanisms. EMBO J 18: 188-197, 1999.

21. Brenner DA, O'Hara M, Angel P, Chojkier M and Karin M: Prolonged activation of jun and collagenase genes by tumour necrosis factor-alpha. Nature 337: 661-663, 1989.

22. Polavarapu R, Gongora MC, Winkles JA and Yepes M: Tumor necrosis factor-like weak inducer of apoptosis increases the permeability of the neurovascular unit through nuclear factor-kappa B pathway activation. J Neurosci 25: 10094-10100, 2005.

23. Thomson EM, Williams A, Yauk CL and Vincent R: Overexpression of tumor necrosis factor- $\alpha$ in the lungs alters immune response, matrix remodeling and repair and maintenance pathways. Am J Pathol 180: 1413-1430, 2012.

24. Moriwaki T, Takagi Y, Sadamasa N, Aoki T, Nozaki K and Hashimoto N: Impaired progression of cerebral aneurysms in interleukin-1beta-deficient mice. Stroke 37: 900-905, 2006.

25. Wang K and Holterman AX: Pathophysiologic role of hepatocyte nuclear factor 6. Cell Signal 24: 9-16, 2012.

26. Jayaraman T, Paget A, Shin YS, Li X, Mayer J, Chaudhry H, Niimi Y, Silane M and Berenstein A: TNF-alpha-mediated inflammation in cerebral aneurysms: A potential link to growth and rupture. Vasc Health Risk Manag 4: 805-817, 2008.

27. Frösen J, Tulamo R, Heikura T, Sammalkorpi S, Niemelä M, Hernesniemi J, Levonen AL, Hörkkö S and Ylä-Herttuala S: Lipid accumulation, lipid oxidation and low plasma levels of acquired antibodies against oxidized lipids associate with degeneration and rupture of the intracranial aneurysm wall. Acta Neuropathol Commun 1: 71, 2013

28. Ruigrok YM, Tan S, Medic J, Rinkel GJ and Wijmenga C: Genes involved in the transforming growth factor beta signalling pathway and the risk of intracranial aneurysms. J Neurol Neurosurg Psychiatry 79: 722-724, 2008.

29. Tan F, Morris PW, Skidgel RA and Erdos EG: Sequencing and cloning of human prolylcarboxypeptidase (angiotensinase C). Similarity to both serine carboxypeptidase and prolylendopeptidase families. J Biol Chem 268: 16631-16638, 1993.

30. Shoja MM, Agutter PS, Tubbs RS, Payner TD, Ghabili K and Cohen-Gadol AA: The role of the renin-angiotensin system in the pathogenesis of intracranial aneurysms. J Renin Angiotensin Aldosterone Syst 12: 262-273, 2011.

31. Dutton A, Young LS and Murray PG: The role of cellular FLICE inhibitory protein (c-FLIP) in the pathogenesis and treatment of cancer. Expert Opin Ther Targets 10: 27-35, 2006.

32. Aoki T, Kataoka H, Morimoto M, Nozaki K and Hashimoto N: Macrophage-derived matrix metalloproteinase- 2 and -9 promote the progression of cerebral aneurysms in rats. Stroke 38: 162-169, 2007.

This work is licensed under a Creative Commons Attribution-NonCommercial-NoDerivatives 4.0 International (CC BY-NC-ND 4.0) License. 